\title{
Práticas de Odontologia em Saúde Coletiva na Estratégia Saúde da Família
}

Edson Lucio da Silva Moura*; Erick Ely Gomes de Oliveira*; Felipe Safh**; Liliane Silva do Nascimento***; Gustavo Antônio Martins Brandão***

* Graduado em Odontologia pela UFPA.

** Coordenador da Atenção da Básica da Secretaria de Saúde de Belém/SESMA

*** Docente Doutor do Departamento de Saúde Coletiva da Faculdade de Odontologia da UFPA

\section{RESUMO}

A Estratégia Saúde da Família no Brasil tem o objetivo de priorizar ações de promoção e proteção à saúde dos indivíduos e da família no âmbito da coletividade e é fundamentada na reorientação e reformulação do processo de trabalho no contexto do Sistema Único de Saúde. Esta estratégia de expansão, qualificação e consolidação da atenção básica é centrada na vigilância à saúde por meio de ações de promoção e recuperação, baseando-se na nova concepção sobre o processo saúde-doença, com atenção voltada para a família e com ações organizadas em território definido. A odontologia insere-se, também, no âmbito da promoção de saúde, de forma que as atividades desenvolvidas pelos profissionais da área não estão adstritas apenas aos tratamentos curativos dos agravos bucais. Este relato de experiência objetiva refletir práticas no sistema de saúde vivenciadas por alunos de Odontologia da Universidade Federal do Pará. O contato com a realidade local tornou os sujeitos mais sensíveis e críticos na sua atuação no Sistema Único de Saúde, além de oportunizar o trabalho em equipe multidisciplinar e consolidar conceitos teóricos da atenção básica em saúde vivenciados na graduação.
Descritores: Odontologia Comunitária. Saúde da Família. Educação Superior

\section{INTRODUÇÃO}

No Brasil, as Diretrizes Curriculares Nacionais (DCN) para a área da saúde explicitam a necessidade de metodologias que favoreçam o processo ensinoaprendizagem e a integração da universidade com os serviços de saúde, de modo a formar profissionais com ênfase na promoção de saúde e prevenção de doenças ${ }^{1}$.

A Lei $n^{\circ} 8.080 / 1990$, que instituiu o Sistema Único de Saúde (SUS) como um sistema de caráter público, formado por uma rede de serviços regionalizada, hierarquizada, com direção única em cada esfera de governo, determinou a observância, dentre outros, dos princípios básicos de universalização, descentralização, integralidade e participação da comunidade, norteando a formação dos profissionais da saúde ${ }^{2}$. A mudança no perfil de formação do cirurgião-dentista tem sido pautada nestes princípios, o que exige 
das instituições de ensino superior a inserção dos alunos nos serviços de saúde e consequente diversificação dos cenários de ensino-aprendizagem ${ }^{3}$.

Diante desses preceitos, surge a necessidade de integrar a universidade e o sistema público de saúde de tal forma que permita diversificar ambientes de aprendizagem, favorecendo a construção de um perfil acadêmico e profissional com competências, habilidades e conteúdos atualizados. Sendo assim, o novo desafio das universidades vem sendo a superação do modelo centrado no diagnóstico e no tratamento para implantação de novas tendências, cuja diretriz deve pautar-se na promoção de saúde e na formação para o $\mathrm{SUS}^{2,3}$.

A Estratégia Saúde da Família (ESF) foi criada em 1994 pelo Ministério da Saúde com o objetivo de priorizar ações de promoção e proteção à saúde dos indivíduos e da família no âmbito da coletividade ${ }^{4}$. Este modelo tem se tornado, nos últimos anos, o principal propulsor da reorientação dos serviços de saúde na atenção básica, além de fortalecer a integração ensino/serviço no Brasil, e aponta para a reformulação do processo de trabalho, centrado na vigilância em saúde, por meio de ações de promoção e recuperação, baseando-se na concepção sobre o processo saúde-doença, com atenção voltada para a família e com ações organizadas em um território definido.

Neste cenário, a Política Nacional de Saúde Bucal (PNSB) estrutura o ensino da Odontologia e dialoga com os projetos pedagógicos quanto à formação do generalista, no enfoque das ações na atenção primária à saúde ${ }^{4}$.

Com base nessa premissa, as Práticas em Saúde Coletiva da Universidade Federal do Pará (UFPA), fundamentadas nas políticas de integração ensino-serviço, propõem-se a realizar a inclusão dos sujeitos em cenários reais e diversos, ampliando sua inserção na sociedade, além de fomentar o senso crítico e a sensibilidade social dos alunos de graduação.

Desse modo, os autores se propõem a discutir o estágio e a integração ensinoserviço em unidades de atenção primária para a formação do cirurgião dentista para o trabalho integrado na ESF.

\section{RELATO DE EXPERIÊNCIA}

Trata-se de estudo observacional, no formato relato de experiência, de integração ensino-serviço do Curso de Odontologia da UFPA em Unidade de Saúde da Família (USF) na cidade de Belém/Pará, Brasil.

A cobertura de equipes de saúde bucal na ESF no município é de $4 \%$, possuindo apenas seis equipes registradas no Cadastro Nacional de Estabelecimentos de Saúde (CNES). A lógica organizacional da gestão distribui os distritos administrativos entre as instituições de ensino. O Distrito Escola da UFPA recebe o nome D'água, sendo o distrito de maior densidade populacional na cidade de Belém/Pará, com aproximadamente 500 mil habitantes, abrangendo cinco bairros com população de conglomerados vulneráveis, áreas de invasão e complexo insular (população ribeirinha). Há duas unidades básicas de saúde no modelo tradicional e somente uma Equipe de Saúde Bucal agregada a duas Equipes de Saúde da Família.

A escolha da USF Condor baseou-se no critério de que a unidade tem a melhor estrutura física, com quatro equipes da saúde da família e possui o território mapeado, atingindo $100 \%$ de cobertura no bairro Condor, além de ter seus profissionais vinculados aos projetos PROPET e PET Vigilância. A unidade foi 
inaugurada em setembro de 2013, embora desde o ano de 2003 fosse cadastrada como Programa de Agentes Comunitários (PACs), tendo implementados os programas Hiperdia, atenção à gestante, planejamento familiar, atenção ao idoso, prevenção e diagnóstico precoce da tuberculose e hanseníase.

Propor a integração ensino-serviço em redes já estruturadas e pactuadas com a gestão local favorece a lógica pedagógica quando consideramos o estágio como a prática de campo que deve proporcionar aos estudantes a transição do mundo acadêmico para o mundo do trabalho ${ }^{5,6}$. Este relato vem refletir outro caminhar de construção desta integração: fazer e inserir a Odontologia onde ela não foi projetada ou ainda não funciona dentro da lógica de gestão local, vivenciando os debates sobre a posição da universidade no fortalecimento do sistema, a reflexão da profissão e a estrutura do sistema de saúde, por meio da atuação conjunta docente, discente e equipes de saúde da família.

As atividades aconteceram de modo programado com as equipes durante uma semana de reunião na unidade. Com planejamento conjunto da equipe mediada pelo docente, uma programação de quatro meses foi definida, com ações semanais, incluindo oficinas, rodas de conversa, palestras, "escovódromos", pré-natal odontológico, visitas domiciliares, ações integradas no Hiperdia e campanhas nacionais (vacinais).

O local de trabalho foi a própria sede da unidade do bairro. Dentre as atividades desenvolvidas, destacou-se a apresentação de uma mesa clínica com variados produtos de higiene bucal disponíveis no mercado (escovas, enxaguantes, dentifrícios, fio e fita dental) de diversas marcas, que aguçou a curiosidade da comunidade e de toda equipe envolvida. Foram realizadas oficinas sobre cárie e doença periodontal, evidenciando a importância da higiene para saúde e como os citados produtos podem auxiliar na prevenção das doenças, além da apresentação das técnicas de escovação com o uso de manequins, sempre buscando a interação com o público e partindo do conhecimento prévio da população, favorecendo $\mathrm{o}$ processo ensinoaprendizagem. $^{7-10}$.

Ao final das atividades foram apresentados métodos alternativos para higienização da cavidade bucal, considerando as condições de vulnerabilidade econômica dos participantes, tais como escovas confeccionadas com palitos de picolé e cabo de bambu, fazendo o uso de bucha vegetal e fio de barbante, fio dental com tiras de sacos plásticos, sacas de transporte de leguminosas, solução de bicarbonato de sódio e água como dentifrício alternativo, entre outras.

De igual modo, as palestras direcionadas aos grupos de risco foram estratégias exitosas, sobretudo a hipertensos e diabéticos (Hiperdia) sobre as doenças sistêmicas e repercussões bucais e viceversa, abordando também a importância do autoexame para a prevenção do câncer de boca.

Por fim, as visitas domiciliares foram previamente agendadas pelos agentes comunitários de saúde que, devidamente capacitados por meio das palestras realizadas, buscavam, no território, famílias classificadas nos grupos de maior risco. As visitas seguiram uma sequência baseada no processo de trabalho: visita de diagnóstico do estado de saúde bucal das famílias (preenchimento das fichas, avaliação das condições de higiene, observação das escovas dentais e planejamento de tratamento dentro das possibilidades da Revista da ABENO • 15(3):52-59, 2015. 
atenção básica), visita pós-planejamento de caso, na qual os alunos atendem em domicílio realizando procedimentos mais simples como raspagens, tratamento restaurador atraumático e exodontias de raízes residuais, distribuição de panfletos educativos e kits de higiene, assim como escovação supervisionada dentro das dependências das residências, avaliando a condição de água e esgoto, bem como armazenamento das escovas dentais.

Ações fora do espaço físico da academia são fundamentais para que os discentes conheçam os cenários reais, onde futuramente atuarão sem supervisão ou apoio dos professores. A universidade tem como responsabilidade social levar assistência às comunidades ao seu redor, prestando serviços e realizando atividades de educação e promoção de saúde, o que corrobora com as atividades realizadas pelos discentes ${ }^{6,11-13}$.

Estas práticas aconteceram em três semestres subsequentes, de março de 2014 a junho de 2015, com ações positivas de impacto direto ao discente em sua formação, à equipe pela integralidade e transversalidade dos temas de saúde com a Odontologia e à população com benefícios diretos ao empoderamento do autocuidado e reconhecimento de problemas e fluxo de resolução na rede de saúde.

Destaca-se que existe impacto relevante na formação acadêmica, devido ao cenário na área da Saúde Coletiva, com desenvolvimento da capacidade de diálogo, respeito aos colegas e tomada de decisões, além da apreensão de aspectos do controle social e sua importância para a gestão da unidade $^{8,10}$.

Sobre a questão social, a maioria dos discentes se mostrou sensibilizada diante dos diversos problemas relatados pela comunidade, apresentando-se envolvidos, engajados, preocupados com a situação local e com vontade de querer colaborar e encaminhar os casos aos serviços especializados. Todos os alunos tiveram a oportunidade de vivenciar os diversos desafios que o SUS propõe para os profissionais da saúde e de entender o quanto é necessário conhecer o sistema para conseguir inserir-se e lutar para melhoria na qualidade do atendimento.

\section{CONSIDERAÇÕES FINAIS}

A realização do estágio com fortalecimento da integração ensino-serviço dentro da ESF teve como principal objetivo a reorganização da prática da atenção ao cuidado integral à saúde bucal sob novas bases, centrando o cuidado à família na tentativa de alcançar melhor qualidade de vida dos brasileiros ${ }^{14}$.

A inserção em cenário de construção constitui desafio bilateral entre a universidade e a gestão, no qual entende-se que as políticas de saúde indutoras de fortalecimento desta integração consolidaram o entendimento da importância da odontologia aos profissionais de outras áreas, que receberam os discentes como agregadores no processo de trabalho, onde o discente é modificado neste processo $^{4,15,16}$.

A prática fortalece a Política do Brasil Sorridente local, pois o discente entende que a saúde bucal é integrante do processo de cuidado na atenção básica. Assim, sua incorporação na estratégia é vista e discutida como possibilidade de romper, a médio e longo prazo, a lógica programática dos modelos tradicionais de ensino nas clínicas escolas, observadas em algumas instituições de ensino superior com os modelos assistenciais em saúde bucal excludentes, baseados no curativismo, tecnicismo e biologicismo. A inserção dos 
alunos de graduação nos cenários vivos de prática corroborara para o processo de formação do novo cirurgião-dentista capaz de trabalhar em equipe e instituir o cuidado em saúde dentro do cotidiano da vida das famílias, uma ação muito além da boca ${ }^{17-19}$.

A mudança e o enfrentamento de desafios da integração ensino-serviço na diversidade dos modelos políticoorganizacionais dos municípios pode e deve ser feita pela universidade, da micro à macrogestão nas pactuações de campos de estágios, dialogando e reconstruindo a aceitação ideológica de docentes e discentes. Uma vez que na contramão da proposição de formação integral do discente, algumas atividades de ensinoaprendizagem fora dos muros das universidades, muitas vezes dependem mais da voluntariedade de alguns docentes do que do apoio institucional e da participação do quadro docente como um todo ${ }^{20-22}$.

Embora o SUS constitua um significativo mercado de trabalho para os profissionais da odontologia, atualmente, sobretudo com a inserção da saúde bucal na ESF, este fato ainda não tem sido suficiente para produzir o impacto esperado sobre o ensino de graduação, com envolvimento do corpo docente nas atividades externas aos muros da universidade e cenários de construção da coletiva da saúde pública $^{8,16,19,22}$.

Sobrinho $^{16}$, em um relato de experiência, observou que a atividade proporcionada aos acadêmicos dos diversos cursos da área da saúde permitiu vivenciar e atuar nas unidades das ESF, contribuindo para a interação acadêmica e atuação transdisciplinar e multiprofissional dos mesmos. A aquisição de novos aprendizados propiciada é extremamente relevante para uma formação diferenciada, o que torna o futuro profissional mais

preparado para enfrentar o mercado de trabalho. Nesse sentido, destaca-se que houve satisfatória integração entre os atores durante o processo de trabalho relatado, tanto no que diz respeito à relação docentediscente, quanto à interação academiaserviço. Deve-se enfocar que, apesar dos avanços alcançados com a proposta de atuação profissional diversificada e interdisciplinar, os desafios mencionados só deverão ser superados após a plena integração entre os cursos das ciências da saúde, de modo a permitir maior flexibilidade e compatibilidade curricular $^{16,21,23}$.

É importante salientar que um bom ensino parte, a princípio, do entusiasmo pessoal do docente, ator desse cenário que, muitas vezes, depende das condições proporcionadas pela instituição de ensino, que, igualmente, deve estar ciente da importância do professor no processo de ensino-aprendizagem e nas atividades de integração ensino-serviço ${ }^{7,13}$.

O cirurgião-dentista graduado deve apresentar formação generalista, humanista, crítica e reflexiva para atuar em todos os níveis de atenção à saúde, baseando-se no rigor técnico e científico, sendo capacitado ao exercício de atividades referentes à saúde bucal da população, pautado em princípios éticos, legais e na compreensão da realidade social, cultural e econômica do seu meio, dirigindo sua atuação para a transformação da realidade em benefício da sociedade ${ }^{1,7,15}$.

Com base nas observações realizadas, verificou-se o quanto as atividades fora do ambiente interno do curso de graduação nos cenários reais da assistência à saúde municipal são determinantes para a formação profissional, pois proporcionam ao discente a oportunidade de conhecer o mundo do trabalho, favorecendo a autonomia em relação à tomada de decisões, Revista da ABENO • 15(3):52-59, 2015. 
desenvolvimento da capacidade de ouvir, trabalho em equipe multiprofissional, habilidade manual, formação humanística, conhecimentos em relação ao sistema vigente no país, capacidade de diálogo, ações de promoção em saúde, capacidade de planejamento de ações em promoção de saúde e com certeza um novo olhar sobre a realidade, a qual vivenciada de perto não pode ficar guardada e sim expandida e compartilhada para o conhecimento de todos, garantindo a articulação ensinopesquisa-extensão.

Assim, compreende-se que a inserção da odontologia nos cenários de práticas fortalece o trabalho multiprofissional e impulsiona o caráter transformador da realidade da comunidade e da gestão dos serviços de saúde, pois ampliou o acesso da população à assistência odontológica, priorizando ações de prevenção e promoção da saúde bucal.

\section{AGRADECIMENTOS}

Agradecemos à Secretaria Municipal de Saúde de Belém e aos servidores da Estratégia Saúde da Família (USF Condor).

\section{REFERÊNCIAS}

1. Diretrizes Curriculares Nacionais do Curso de Graduação em Odontologia. Resolução CNE/CES no 3, de 19 de fevereiro de 2002.

2. BRASIL. Lei $\mathrm{n}^{\circ} 8.080$, de 19 de setembro de 1990. Dispõe sobre as condições para a promoção e recuperação da saúde, a organização e o funcionamento dos serviços correspondentes e dá outras providências. Diário Oficial [da] República Federativa do Brasil, Brasília, 19 set. 1990.
3. Pereira CRS, Patrício AAR, Araújo FAC; Lucena EES, Lima KC, Roncalli AG. Impacto da Estratégia Saúde da Família com equipe de saúde bucal sobre a utilização de serviços odontológicos. Cad Saúde Pública 2009; 25(5):985-96.

4. Silva BS, Caldarelli PG. O PET-Saúde em consonância com as Diretrizes Curriculares Nacionais de Odontologia no desenvolvimento de competências profissionais: relato de experiência, Rev ABENO 2013; 13(2):34-41.

5. Ministério da Saúde. Portaria $n^{\circ} .74$. Reajusta os valores dos incentivos financeiros às ações de saúde bucal no âmbito do Programa Saúde da Família, inclui procedimento de moldagem para prótese e dá outras providências. Diário Oficial da União 2004; 22 jan. 2004.

6. Botazzo C, Fonseca GS, Junqueira SR. Modelo lógico-ideal para o estágio curricular supervisionado: a educação pelo trabalho na formação Odontológica. Rev ABENO 2013; 15(2):2-11.

7. Freire P. A Pedagogia do Oprimido. Rio de Janeiro: Paz e Terra, 1981.

8. Galassi MAS, Barbin EL, Spanó JCE, Melo JAJ, Tortamano N, Carvalho ACP. Atividades extramuros como estratégia viável no processo ensinoaprendizagem: Uma variação do ambiente ensino/aprendizagem valiosa tanto pelo contato do aluno com a comunidade, quanto pela grande interação entre professor e aluno. Rev ABENO 2006;6(1):66-9.

9. Júnior ACRA. Paisagem antropogenética e a dinâmica do meio físico na porção sul da cidade de Belém (PA). Ateliê Geográfico 2013; 7(2):71-96.

10. Moimaz SAS, Santos RR, Saliba O, Borges HC, Garbin CAS, Ancieri RM, Revista da ABENO • 15(3):52-59, 2015. 
Saliba NA. A experiência da saúde coletiva na formação profissional: retrato da extensão universitária. Rev Ciênc Ext 2013; 9(2):152-66.

11. Toassi RFC, Baumgarten A, Warmling CM, Rossoni E, Rosa AR, Slavutzky SMB. Teaching at primary healthcare services within the Brazilian national health system (SUS) in Brazilian healthcare professionals' training. Interface 2013; 17(45):385-92.

12. Oliveira M L, Coelho TC. A percepção de acadêmicos de odontologia sobre o PET-Saúde UFMS/SESAU, Campo Grande/MS. Rev ABENO 2011; 11(1):76-80.

13. Oliveira MS. O papel dos profissionais de saúde na formação acadêmica. Olho Mágico 2013; 10 (2):37-9.

14. Souza TMS, Roncalli AG. Saúde bucal no Programa Saúde da Família: uma avaliação do modelo assistencial. Cad Saúde Pública 2007; 23(11):2727-39.

15. Sobrinho TAO, Medeiros CPP, Maia MR, Reis TC, Miranda LP, Costa PF. Integração acadêmica e multiprofissional no Pet-Saúde: experiências e desafios. Rev ABENO 2011; 11(1):39-42.

16. Saliba NA, Saliba O, Moimaz SAS, Garbin CAS, Arcieri RM, Lolli LF. Integração ensino-serviço e impacto social em cinquenta anos de história de saúde pública na Faculdade de Odontologia da Universidade Estadual Paulista Júlio de Mesquita Filho. RGO 2009; 57(4):459-65.

17. Morita MC, kriger L. Mudanças no curso de odontologia e a interação com o SUS. Rev ABENO 2004; 4(1):17-21.

18. Ministério da Saúde. Portaria no. 267. Aprova as Normas e Diretrizes de Inclusão da Saúde Bucal na Estratégia do Programa de Saúde da Família
(PSF). Diário Oficial da União 2001; 7 mar

19. Moimaz SAS, Saliba NA, Zina LG, Saliba O, Garbin CAS. Prática de ensino-aprendizagem com bases em cenários reais. Interface 2010; 14(32):69-79.

20. Carvalho BR, Costa TBC, Gomes MJ, Santos KT, Guerra SMG. Formação docente em Odontologia no Brasil: sugestões de mudanças após as Diretrizes Curriculares Nacionais. Rev Bras Pesq Saúde 2010; 12(4): 39-44.

21. Raldi DP, Malheiros CF, Fróis IM, Lage-Marques JL. O papel do professor no contexto educacional sob o ponto de vista dos alunos. Rev ABENO 2003; 3(1):15-23.

22. Moimaz SAS, Saliba NA, Garbin CAS, Zina LG, Furtado JF, Amorim JA. Serviço extramuro odontológico: impacto na formação profissional. Pesqui Bras Odontopediatria Clín Integr 2004; 4(1):53-7.

23. Calado GS. A inserção da equipe de saúde bucal no Programa de Saúde da Família: principais avanços e desafios [Dissertação de Mestrado]. Rio de Janeiro: Escola Nacional de Saúde Pública, Fundação Oswaldo Cruz; 2002.

\section{ABSTRACT \\ Collective Health Dental Practices in the Family Health Strategy}

The Family Health Strategy (FHS Estratégia Saúde da Família/ESF) in Brazil aims to prioritize promotion and protection of the health of individuals and families within the community. It is based on the reorientation and reformulation of the work process in the context of the Single Health System (SHS). The expansion strategy, qualifications and primary care consolidation are centered on health care through promotion and recovery actions, 
based on the new conception of the healthdisease process, with attention focused on family and organized actions in territory defined. In this field, dentistry is inserted also in health promotion, so that the activities developed by professionals are not restrict only to the curative treatment of dental caries. This experience report aims to reflect about practices in health care experienced by dental students at Federal University of Pará. The contact with the local reality became the most sensitive and critical subject in its operations in the National Health System, as well as provide the opportunity for multidisciplinary work and consolidate theoretical concepts of primary health care team experienced on graduation.
Descriptors: Community Dentistry. Family Health. Education, Higher.

Correspondência para:

Profa. Dra. Liliane Silva do Nascimento

e-mail: lilianenascimento2001@ gmail.com

Tv Barão do Triunfo, 3508/2201

66095-050 - Belém/PA 